\title{
Control basado en campos potenciales para un vehículo autónomo usando realimentación visual
}

\author{
Oscar González-Miranda, Santos M. Orozco-Soto, Juan M. Ibarra-Zannatha \\ CINVESTAV, Departamento de Control Automático, México \\ \{ogonzalez, sorozco\}@ctrl.cinvestav.mx, jibarra@cinvestav.mx
}

\begin{abstract}
Resumen. En este trabajo se desarrolló un controlador para conducir de forma autónoma, al vehículo a escala 1:10 AutoNOMOS, desarrollado por la Freie Universität Berlin. El vehículo se condujo por una carretera blanca, dibujada sobre un fondo negro, sin obstáculos y a una velocidad de crucero constante de $0.6 \frac{\mathrm{m}}{\mathrm{s}}$. Este controlador usa la información visual de la cámara y un enfoque similar al usado en el Control Automático Basado en Campos Potenciales; para regular el ángulo de dirección $u(t)$ del automóvil. Se probó su desempeño en el simulador Gazebo y se demostró que el vehículo podía mantenerse dentro de su carril durante todo el recorrido.
\end{abstract}

Palabras clave: vehículo autónomo, control de dirección, navegación reactiva, control basado en campos potenciales, control con realimentación visual.

\section{Potential-Field Based Control for Autonomous Vehicle Using Visual Feedback}

\begin{abstract}
This paper presents the design and implementation of a con-trol strategy for autonomous driving of the AutoNOMOS, a 1:10 scaledvehicle developed by the Freie Universität Berlin. The autonomous nav-igation problem is to drive the vehicle within a lane of a 2-way road,which is white painted over black ground, without obstacles but withcrossroads, and additionally, keeping a constant navigation speed of $0.6 \mathrm{~m} / \mathrm{s}$. The presented controller uses visual feedback and a field potential-based approach in order to supply a steering angleu(t) for the auto-mobile. The performance of the controller was tested using the Gazebosimulator, and it was proved that the vehicle is capable to drive withinits corresponding lane during the whole road.
\end{abstract}

Keywords: autonomous vehicle, steering control-reactive navigation, potential field control, visual feedback control.

\section{Introducción}

En los últimos años las grandes empresas del sector automotriz han llevado a cabo una competencia para conseguir vehículos completamente autónomos y 
aunque algunas ya han logrado introducir autos de este tipo en el mercado; aún existen problemas que exigen cierto nivel de robustez para conseguir una conducción más segura y confortable. Esta clase de problemas se han vuelto muy importantes en la investigación. Varias universidades de México están incursionando en esta clase de tecnologías gracias a las donaciones hechas por el Dr. Raúl Rojas de la Freie Universität Berlin [7]. Desde el 2016, una treintena de Instituciones de Educación Superior, organizadas por el Instituto Politécnico Nacional, han recibido prototipos de vehículos autónomos a escala 1:10. Este trabajo se enfoca en este vehículo en particular.

El problema de la condución autónoma, se suele separar en otros más específicos: Planeación de trayectorias[6,7,8], el cual consiste en elegir una trayectoria que sea óptima (bajo algún criterio) para el vehículo; seguimiento de trayectorias $[4,13]$, el cual consiste en diseñar un controlador que le permita al automóvil mantenerse en la trayectoria deseada mientras avanza; mapeo del medio que rodea al auto a partir de la información sensorial [15]; realización de maniobras como la evasión de obstáculos o el revase de otros vehículos [12]; entre otros.

En particular, conducir un vehículo tipo Ackerman manteniéndolo en su carril se puede definir como el problema de calcular el ángulo de dirección del automóvil, para dar seguimiento a una trayectoria de referencia; mantieniendo el auto en el centro del camino y con una orientación apropiada [14]. Este problema se ha resuelto modelándolo de diferentes formas y estableciendo controladores, tanto lineales como no lineales $[1,2,7,11,14]$; sin embargo, existen fuerzas externas como la fricción estática, la fuerza debida a la interacción del frente con el aire o la fuerza del viento sobre el centro aerodinámico; que hacen dificil la identificación de los parámetros del modelo y por lo tanto las estrategias de control pierden robustez en ambientes externos [9].

En este trabajo se desarrolló una ley de control para conducir el AutoNOMOS por una carretera de dos carriles, con un par de cruces y sin obstáculos; a una velocidad constante de $0.6 \frac{\mathrm{m}}{\mathrm{s}}$. La realimentación visual se consiguió usando la cámara RGBD a bordo del vehículo. A las imágenes obtenidas se les aplicó una corrección de perspectiva para calcular la distancia entre el centro del vehículo y la línea del carril derecho. La estrategia de control utilizada es una técnica basada en Campos Potenciales, diseñada para atraer al vehículo al centro del carril y mantener el vehículo en la orientación deseada.

El controlador se implementó en el sistema operativo para robots (ROS) y es ejecutado en tiempo real. Se probó su desempeño en el simulador Gazebo desarrollado por la Freie Universität Berlin. Este trabajo está organizado de la siguiente manera: la sección 2 presenta la descripción del hardware. El diseño del sistema de control se detalla en la Sección 3. La sección 4 describe las simulaciones y sus resultados. Finalmente, las conclusiones se exponen en la Sección 5. 


\section{Descripción del hardware}

El vehículo autónomo usado en este trabajo es el AutoNOMOS, el cual es un automóvil tipo Ackerman a escala 1:10 que mide $40 \mathrm{~cm}$ de largo y $20 \mathrm{~cm}$ de ancho. Utiliza un motor sin escobillas de 24 Watts para mover las ruedas y una batería Li-Po de $14.8 V$ de 4 celdas. El ángulo de dirección es controlado por un servomotor de $5 \mathrm{~V}$. A bordo se encuentra una computadora Odroid XU4 con el sistema operativo Ubuntu 16.04 y la distribución ROS Indigo instalada. El auto también está equipado con sensores como el sensor inercial MPU6050, sensores de efecto Hall embebidos en el motor sin escobillas, una cámara fish-eye, una cámara RGBD y un LIDAR infrarrojo. La figura 1 muestra al AutoNOMOS y algunos de su dispositivos a bordo.

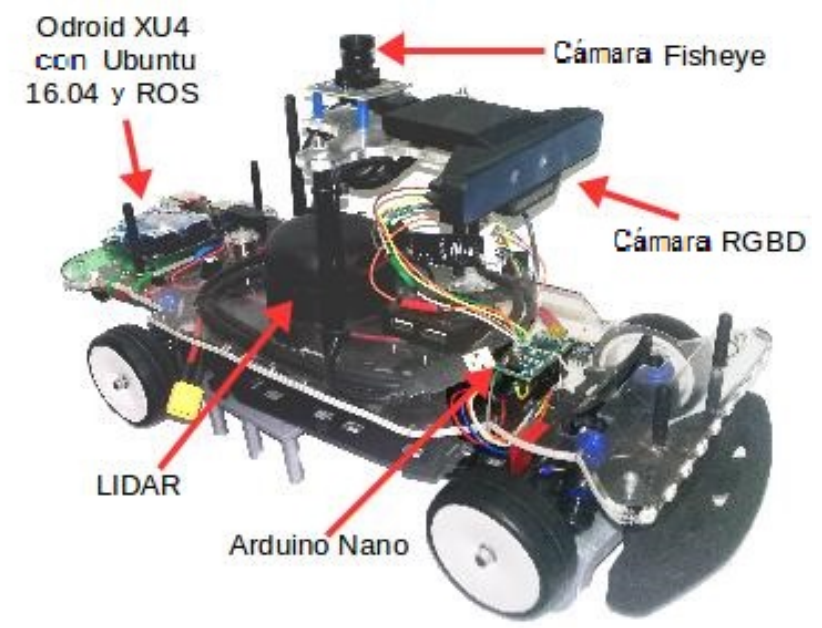

Fig. 1. Vehículo AutoNOMOS [3].

\section{Desarrollo del sistema de Control}

\subsection{Definición del problema}

El objetivo de control es poder conducir el auto a través de una carretera blanca dibujada sobre un fondo negro, con cruces, sin obstáculos, sin conocimiento a priori del mapa y sin salirse de su carril. Para llevar a cabo este objetivo se usa la cámara RGBD para reconocer la distancia $x_{1}$ (en centímetros), desde la línea del lado derecho del camino, hasta el centro de la parte inferior de la imagen denotado como $\{N\}$; tal y como se muestra en la figura 2. Esta imagen 
presenta la vista desde arriba del camino; obtenida a través de la corrección de perspectiva de la imagen tomada por la cámara RGBD. Nótese que el punto $\{N\}$ representa la posición del vehículo y más adelante es usado como marco referencial de navegación. Por otro lado, $x_{2}$ también es la distancia entre el centro de la imagen hasta la línea de la derecha del camino; pero $15 \mathrm{~cm}$ por encima de $\{N\}$. Independientemente de la forma del camino si el vehículo está en el centro de su carril entonces $x_{1}=x^{d}=20 \mathrm{~cm}$. Así pues, las variables de control en este sistema son: la velocidad de cruzero del vehículo $v$ y el ángulo de dirección $u$ del mismo. Por simplicidad se mantuvo constante $v=0,6 \frac{\mathrm{m}}{\mathrm{s}}$. Por lo tanto, de modo general podemos decir que:

Dado un conjunto arbitrario de distancias $X_{1} \subset \mathbb{R}^{+}$conocido como carretera, se conduce al auto con un ángulo de dirección $u \mid x_{1} \in X_{1} \rightarrow x^{d}, x^{d}=20 \mathrm{~cm}$.

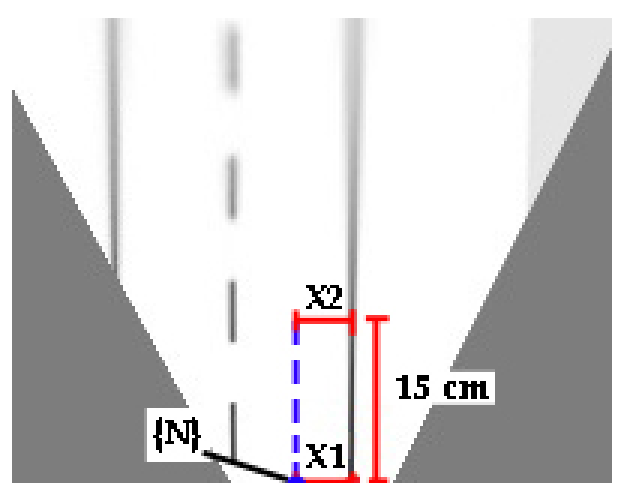

Fig. 2. Fragmento de la homografía de la carretera por la que circula el vehículo autónomo, cuando este se encuentra en el centro del carril derecho. Completa tiene un ancho de $200 \mathrm{~cm}$ y un largo de $300 \mathrm{~cm}$. Cada carril mide $40 \mathrm{~cm}$.

\subsection{Realimentación visual}

Para controlar ángulo de dirección del auto $u$, primero se obtuvo una imagen RGB capturada por la cámara RGBD (figura 3.a) y se recortó el fondo para que sólo se muestre la carretera. Después se transformó la imagen a escala de grises y se hizo la corrección de la perspectiva para tener una imagen del camino vista desde arriba (3.b). Este mapeo se obtiene usando la transformación:

$$
\lambda\left(\begin{array}{l}
x \\
y \\
1
\end{array}\right)=H^{-1}\left(\begin{array}{l}
u \\
v \\
1
\end{array}\right) .
$$

De este modo cada pixel en la posición $(u, v)$ de la imagen, se mapea a las coordenadas $(x, y)$ en el suelo (medidas en centímetros); $\lambda$ es un factor de escala 
diferente para cada pixel y a $H$ se conoce como la matriz de homografía de la cámara [5]. En este caso se calculó que:

$$
H=\left(\begin{array}{ccc}
-7,90 \times 10^{-2} & -4,19 \times 10^{-1} & 1,28 \times 10^{2} \\
1,00 \times 10^{-2} & -1,46 & 4,00 \times 10^{2} \\
0 & -4,15 \times 10^{-3} & 1
\end{array}\right) .
$$

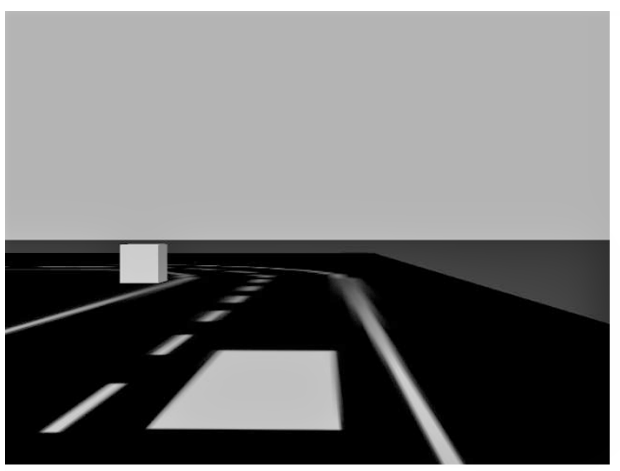

(a)

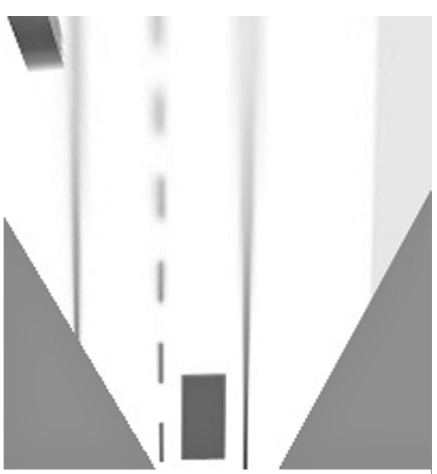

(b)

Fig. 3. Corrección de la perspectiva de la cámara usando la matriz de homografía inversa. Los colores se invierten de (a) a (b) para permitir una mejor visualización. Las esquinas del rectángulo que está dibujado en el suelo se usaron para calcular la matriz de homografía $H$ de la cámara.

Suponiendo que la imagen tiene una resolución de $p \times q$ y dada una matriz $J \in(\mathbb{N} \cup\{0\})^{3 \times n}$ que representa la posición de los $n$ pixeles de la imagen; podemos definir $M$ como:

$$
M=\frac{1}{\lambda} H^{-1} J, \quad M \in \mathbb{R}^{3 \times n} .
$$

Así pues, aplicando un algoritmo de búsqueda dentro de una región de interés $M_{R O I} \subset M$, la distancia $x_{1}$ puede ser calculada usando la expresión:

$$
x_{1}=M\left(\frac{p}{2}, q\right)-M_{R O I}(j, q),
$$

donde $M\left(\frac{p}{2}, q\right)$ es el pixel que se encuentra en el centro de la parte inferior de la imagen (marco referencial de navegación) y $M_{R O I}(j, q)$ es el $j$-ésimo pixel cuya intensidad sobrepasa cierto umbral $\delta$. Por otro lado, $x_{2}$ puede ser calculado como:

$$
x_{2}=M\left(\frac{p}{2}, q\right)-M_{R O I}(j, q-\epsilon),
$$

donde $\epsilon>0$ es un parámetro que depende de la velocidad del vehículo y los FPS de la cámara. 


\subsection{Control basado en Campos Potenciales}

Para determinar el valor de una variable auxiliar de control $\theta$ se usa un enfoque similar al Control Automático de Sistemas Usando Campos Potenciales [10]; es decir, se le asignan potenciales atractivos y repulsivos a ciertos puntos sobre la homografía, se calcula la "fuerzaresultante sobre el vehículo y se determina la dirección que debe tomar. En la figura 4, a los puntos $\overline{r_{1}}$ y $\overline{r_{2}}$ (ambos medidos desde $\left.\{N\}\right)$ se les asignan los potenciales $\frac{K_{0}}{r_{1}} \hat{r}_{1}$ y $-\frac{K_{0}}{r_{2}} \hat{r}_{2}$, respectivamente. $K_{0}$ es un parámetro arbitrario que se simplifica más adelante. Los vectores $\hat{r}_{1}=\cos \left(\theta_{1}\right) \hat{\imath}+\sin \left(\theta_{1}\right) \hat{\jmath} \mathrm{y} \hat{r}_{2}=\cos \left(\theta_{2}\right) \hat{\imath}+\sin \left(\theta_{2}\right) \hat{\jmath}$ son unitarios con componentes vertical $\hat{\jmath}$ y horizontal $\hat{\imath}$; mientras que:

$$
\begin{aligned}
& r_{1}=\sqrt{15^{5}+x_{2}^{2}} \quad y \quad r_{2}=\sqrt{15^{5}+\left(x_{2}-20\right)^{2}}, \\
& \theta_{1}=\arctan \left(\frac{15}{x_{2}}\right) \quad y \quad \theta_{2}=\arctan \left(\frac{15}{x_{2}-20}\right) \text {, }
\end{aligned}
$$

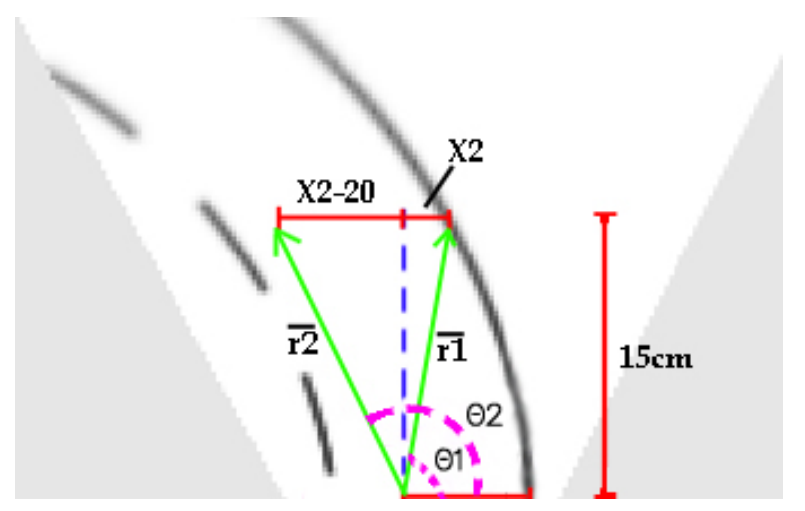

Fig. 4. Obtención de la variable $\theta$ mediante Campos Potenciales.

La dirección vertical $\hat{\jmath}$ siempre es la dirección de avance del vehículo; asi que, para que el vehículo se mueva hacia adelante se supone que sobre él actúa una fuerza $\overrightarrow{F_{1}}=K_{1} \hat{\jmath}$. Por lo tanto, la fuerza resultante en el vehículo esta dada por la ecuación:

$$
\vec{F}=K_{1} \hat{\jmath}+\frac{K_{0}}{r_{1}^{2}} \hat{r}_{1}-\frac{K_{0}}{r_{2}^{2}} \hat{r}_{2} .
$$

De aquí se puede deducir que la dirección del vehículo, medida desde la vertical, esta dada por:

$$
\theta=90-\arctan \left(\frac{K r_{1}^{2} r_{2}^{2}+r_{2}^{2} \sin \left(\theta_{1}\right)+r_{1}^{2} \sin \left(\theta_{2}\right)}{r_{2}^{2} \cos \left(\theta_{1}\right)+r_{1}^{2} \cos \left(\theta_{2}\right)}\right) \quad \text { con }: \quad K=\frac{K_{1}}{K_{0}} .
$$

El signo de $\theta$ determinará si el giro es hacia la izquierda o hacia la derecha; mientras que $K$ es un parámetro que se ajusta experimentalmente. 


\subsection{Ley de control}

El diagrama de bloques de la figura 5 representa al sistema que se desea controlar. El tópico de ROS que conduce el auto está programado de tal modo que, si el ángulo de dirección $u$ cumple que $0 \leq u<90$, el vehículo girará a la izquierda; por otro lado si $90 \leq u<180$ el giro es hacia la derecha. Por lo tanto, se usa como ley de control:

$$
u=90+K_{x} e_{x}+K_{\theta} \theta
$$

donde $e_{x}=x^{d}-x_{1}$ es el error de posición del vehículo cuando este se aleja del centro de su carril, $\theta$ es una variable relacionada con la forma del camino y $K_{x}$ y $K_{\theta}$ son ganancias que se ajustan de acuerdo al desempeño del controlador.

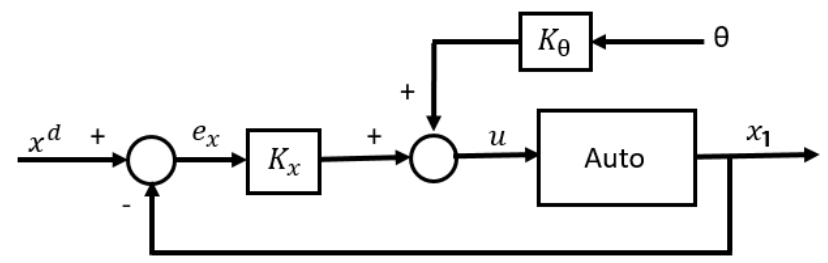

Fig. 5. Diagrama de bloques del sistema.

\section{Simulaciones y resultados}

Para probar el desempeño del controlador de la ecuación (10) con $\theta$ como en la ecuación (9), se le asignaron los valores a los parámetros: $K_{x}=3,0, K_{\theta}=0,25$ y $K=0,01 ;$ y se mantuvo una velocidad de crucero constante de $0.6 \frac{\mathrm{m}}{\mathrm{s}}$. La ley de control se programó como un nodo en ROS y las pruebas se hicieron en el simulador Gazebo con una carretera como la mostrada en la figura 6. La carretera tiene un ancho de $80 \mathrm{~cm}$ por lo que cada carril mide $40 \mathrm{~cm}$. Todas las curvas de la pista tienen un radio externo de $1.8 \mathrm{~m}$ y un radio interno de $1.0 \mathrm{~m}$. 
Oscar González-Miranda, Santos M. Orozco-Soto, Juan M. Ibarra-Zannatha

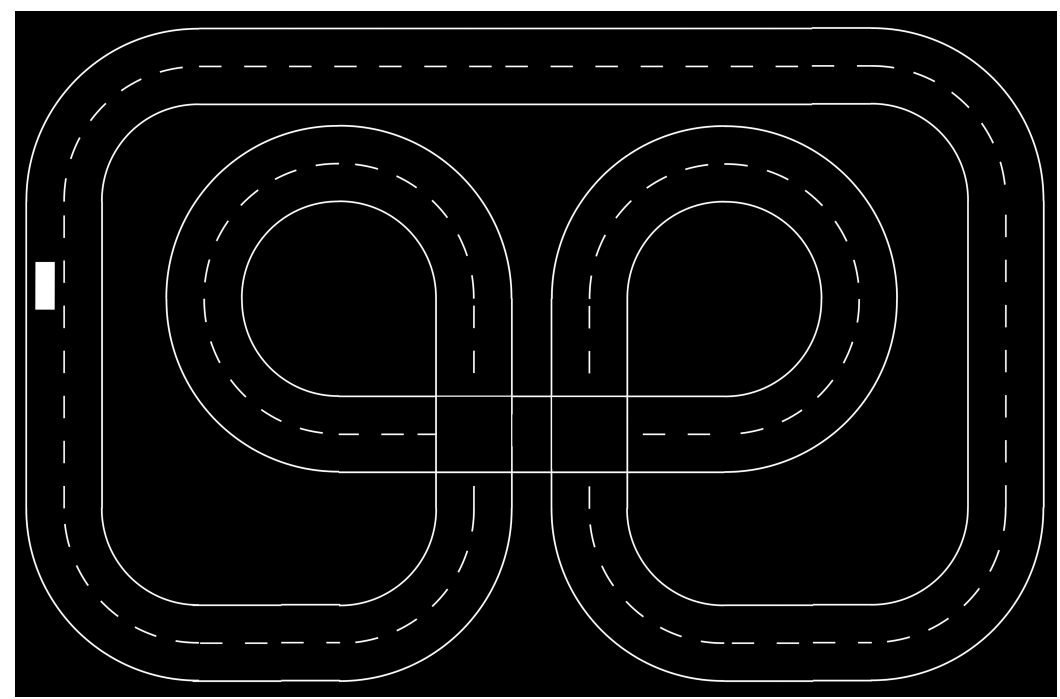

Fig. 6. Pista por la que se condujo el AutoNOMOS en el simulador de Gazebo.

La gráfica de la figura 7 muestra el cambio en el tiempo del error de posición del vehículo; mientras que la gráfica de la figura 8 muestra el ángulo de dirección del mismo. Como se puede observar, el error de posición aumenta cuando el vehículo toma una vuelta y disminuye hasta casi cero mientras el camino es recto. Dado que $-20 \leq e_{x} \leq 20$ en todo momento, entonces el vehículo se mantuvo dentro de su carril durante todo el recorrido.

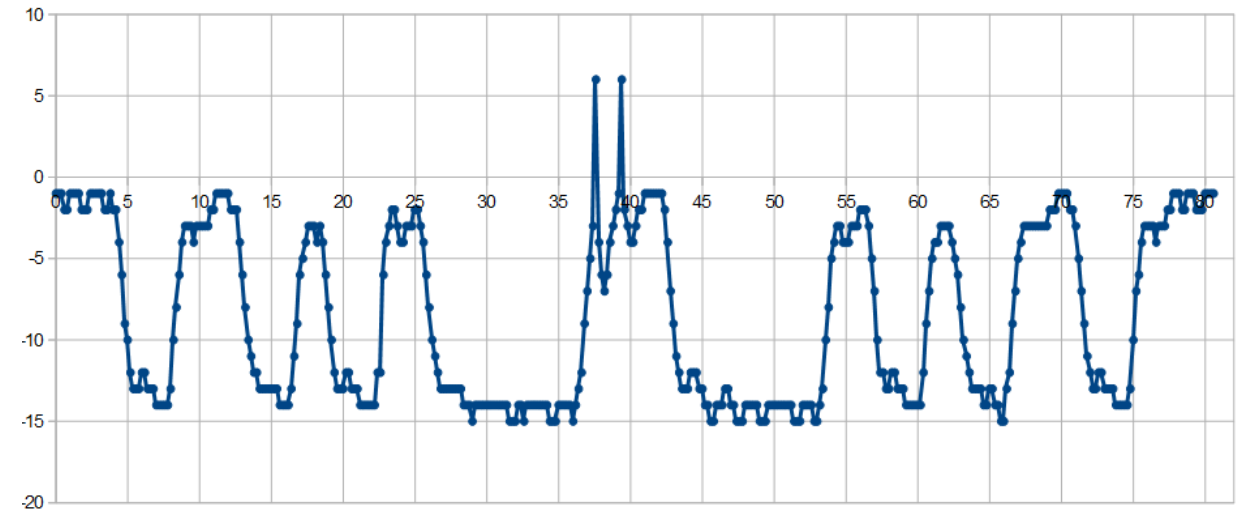

Fig. 7. Evolución del error $e_{x}$ en el tiempo. Si se cumple que $-20 \leq e_{x} \leq 20$ el automóvil se mantendrá dentro de su carril. Esta cantidad está medida en $\mathrm{cm}$. 


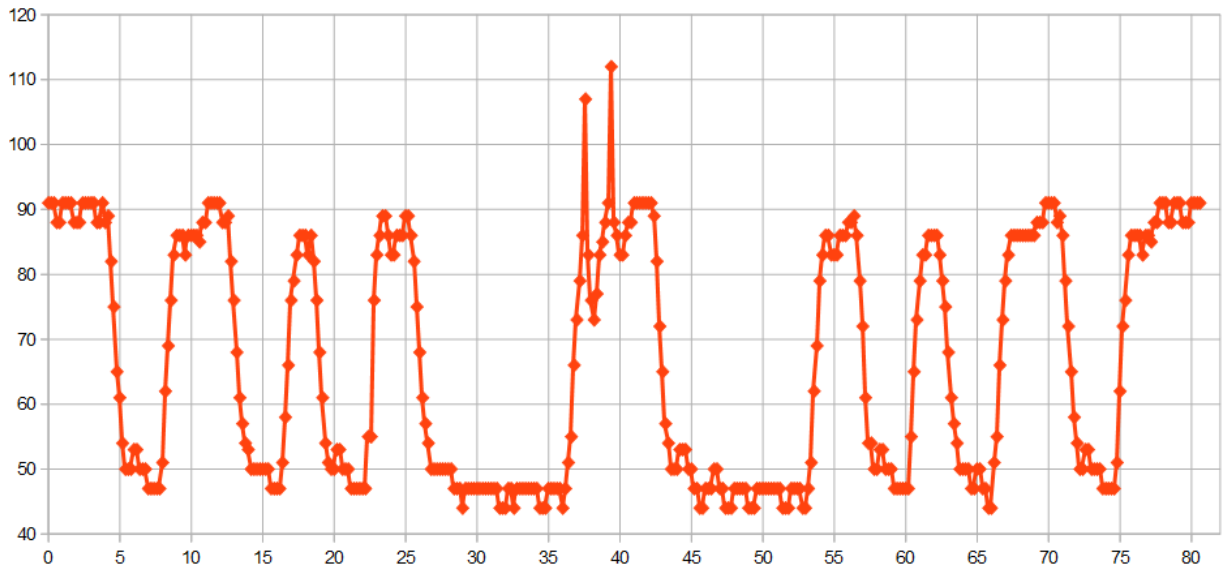

Fig. 8. Cambio del ángulo de dirección $u(t)$ en el tiempo. Este ángulo está medido en grados.

\section{Conclusiones}

A partir de las gráficas de las figuras 7 y 8 podemos concluir que el término que más peso tiene en la ecuación 10 es $K_{x} e_{x}$; pues es el término que modula el ángulo $u(t)$ cuando el vehículo se sale de su carril. Por otro lado, se observó que el término $K_{\theta} \theta$ mejora el desempeño del vehículo.

Así pues el controlador diseñado fue lo suficientemente robusto como para mantener el vehículo dentro de su carril en todo momento y por lo tanto, la metodología empleada ofrece una solución sencilla y fácil de implementar, al problema de la conducción autónoma de un vehículo tipo Ackerman; sin embargo, dicha robustez está limitada a la condición inicial de que $x_{1}(0) \mid e_{x}(0) \in[0,28]$; de otro modo, se tomará mucho tiempo alcanzar el objetivo de control.

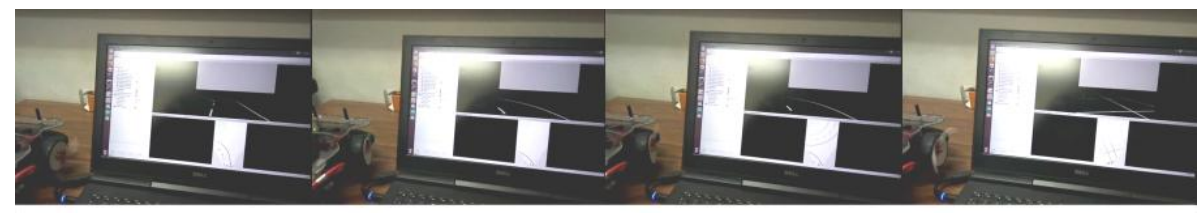

Fig. 9. Capturas del vehículo real conduciéndose por la pista virtual del simulador. Video disponible en: https://www . youtube.com/watch?v=hiniVFp3mio

Aunque aún no se tienen resultados experimentales, se han hecho pruebas con el vehículo AutoNOMOS como la que se muestra en la figura 9. Allí se puede observar cómo el auto cambia su ángulo de dirección adecuadamente; de acuerdo 
a las imágenes de la cámara del simulador. En trabajos futuros se conducirá el vehículo real por una pista blanca con fondo negro.

\section{Referencias}

1. Ackermann, J., Guldner, J., Sienel, W., Steinhauser, R., Utkin, V.I.: Linear and nonlinear controller design for robust automatic steering. IEEE Transactions on Control Systems Technology 3(1), 132-143 (1995)

2. Ackermann, J., Sienel, W.: Robust control for automatic steering. In: 1990 American Control Conference. pp. 795-800. IEEE (1990)

3. Campbell, S.F.: Steering control of an autonomous ground vehicle with application to the DARPA urban challenge. Ph.D. thesis, Massachusetts Institute of Technology (2007)

4. Corke, P.: Robotics, vision and control: fundamental algorithms In MATLABß second, completely revised, vol. 118. Springer (2017)

5. Freie Universität Berlin: AutoNOMOS Model. https://github.com/ AutoModelCar/AutoModelCarWiki/wiki (2016), [Online; accessed 15-April-2019]

6. Hernandez Beleno, R.D., Bernardes Vítor, G., Vaqueiro Ferreira, J., Siqueira Meirelles, P.: Planeación y seguimiento de trayectorias de un vehículo terrestre con base en el control de dirección en un ambiente real. Scientia et technica 19(4) (2014)

7. Ibarra Zannatha1, J.M., Vera Bustamante, P., Orozco Soto, S.M., Cureno Ramírez, A., de la Rosa Picasso1, R., Ramírez Morales, L.E.: Generacion de trayectorias para vehículos autónomos (2018)

8. Katrakazas, C., Quddus, M., Chen, W.H., Deka, L.: Real time motion planning methods for autonomous on-road driving: State of the art and future research directions. Transportation Research Part C: Emerging Technologies 60, 416-442 (2015)

9. Kodagoda, K., Wijesoma, W.S., Teoh, E.K.: Fuzzy speed and steering control of an agv. IEEE Transactions on control systems technology 10(1), 112-120 (2002)

10. Lewis, F.L., Stingu, E., TTXYVVVRFFRX, Y.: Potential fields in cooperative motion control and formations (2013)

11. Marino, R., Scalzi, S., Netto, M.: Nested pid steering control for lane keeping in autonomous vehicles. Control Engineering Practice 19(12), 1459-1467 (2011)

12. Rastelli, J.P., De Pedro, T., Santos, M.: Controladores borrosos para la dirección de vehículos autónomos en maniobras dentro de entornos urbanos. In: XVI Congreso Espanol sobre Tecnologías y Lógica Fuzzy (2012)

13. Snider, J.M., et al.: Automatic steering methods for autonomous automobile path tracking. Robotics Institute, Pittsburgh, PA, Tech. Rep. CMU RITR 0908 (2009)

14. Sotelo, M.A.: Lateral control strategy for autonomous steering of ackerman-like vehicles. Robotics and Autonomous Systems 45(3-4), 223-233 (2003)

15. Thrun, S., Montemerlo, M., Dahlkamp, H., Stavens, D., Aron, A., Diebel, J., Fong, P., Gale, J., Halpenny, M., Hoffmann, G., et al.: Stanley: The robot that won the darpa grand challenge. Journal of field Robotics 23(9), 661-692 (2006) 